\title{
Noise Prediction of Centrifugal Fan Based on Improved Nenral Network
}

\author{
Bo Long ${ }^{1, a}$, Shuxia Jiang ${ }^{1, b^{*}}$, Changwei Zhang ${ }^{1, \mathrm{c}}$ and Wen Liu ${ }^{1, \mathrm{~d}}$ \\ ${ }^{1}$ School of Mechanical and Eletrical Engineering, Central South University of Forestry and Technology, Changsha, Hunan, China
}

\begin{abstract}
In order to solve the problems that the prediction accuracy of the traditional centrifugal fan is low and the cost is high, a noise prediction model for centrifugal fan based on improved particle swarm optimization (IPSO) optimized BP neural network was presented. The initial weights and thresholds of BP neural network were optimized by using IPSO. The 17 parameters were collected by the liancheng company and be used to establish the regression equation to obtain the standard regression coefficient. The importance of the fan parameters was ranked and four key characteristic parameters were determined as input values by the optimization algorithm to build the IPSO-BP centrifugal fan noise prediction model. After comparative study, IPSO-BP model has better prediction effect than PSO-BP model and BP model, and the prediction error is only $0.97 \%$. The research shows that IPSO-BP model can effectively shorten the fan design period and save the design cost.
\end{abstract}

\section{Introduction}

As a populous country, China's demand for metal, coal, steel, electricity and other energy is increasing, along with the mining of energy, but also led to the expansion of the fan market, so the requirements for the performance of the fan is also getting higher and higher. In recent years, foreign experts and scholars have carried out a series of studies on the performance improvement of the fan, and completed the improvement of the efficiency of the fan and the reduction of the noise of the fan through its optimization design. With the increasing awareness of environmental protection, people pay more and more attention to the harm of noise of centrifugal fans, and noise has gradually become an important indicator to judge the performance of centrifugal fans. In order to realize the noise reduction design of the fan, shorten the design and manufacturing cycle, and save the development cost, it is essential to estimate the noise of the fan.

Yao Jingyu ${ }^{[1]}$ predicted the aerodynamic noise of the axial flow fan by combining BP neural network and numerical simulation, and then verified through the test set that the loss value of the noise prediction method by combining neural network with numerical simulation was 0. $255 \%$, indicating the feasibility of this method. Wei $\mathrm{Zhen}^{[2]}$ et al. used Elman neural network to predict wind farm noise, and the simulation results showed that the model could fit well, and the prediction results were in line with the actual situation, which could provide a certain reference for wind farm noise prediction. Hua Chunlei ${ }^{[3]}$ et al. took automobile as the research object and established the prediction model of interior noise. The simulation results showed that the BP neural network could well reflect the variation trend of noise, which was basically consistent with the actual trend, and had a strong reference significance for noise control. Taking CPU as the research object, Li Yaguang ${ }^{[4]}$ used BP neural network to predict $\mathrm{CPU}$ voltage noise, and the prediction accuracy was $94.5 \%$, which met the requirement of CPU voltage noise prediction. $\mathrm{Li} \mathrm{Hui}^{[5]}$ et al. combined Lighthill acoustic analogy theory and BP neural network to predict the external aerodynamic noise of high-speed trains, and the predicted results were in line with the test results, reducing the complexity of aerodynamic noise calculation. Cheng Jing ${ }^{[6]}$ et al. predicted fan noise by combining regression analysis and BP neural network, and applied the model to the actual test of a wind airport in Xinjiang, which achieved good results, but the single BP neural network was unstable. Based on the studies of scholars, it is known that neural network can be widely used in noise prediction, and the prediction accuracy of noise prediction by using neural network is high. The variation trend of predicted noise is basically consistent with the actual trend, so it is feasible to use BP neural network in the prediction of centrifugal fan noise.

\section{Noise Prediction Based on Improved Nenral Network}

\subsection{Data source and preprocessing}

All data in this paper are collected by Zhuzhou Liancheng Group Holding Co, Ltd, a total of 400 types of fans, Each fan includes 17 parameters, including flow rate, total pressure, rotational speed, power, efficiency, volute form, impeller diameter, impeller inlet diameter, blade inlet

\footnotetext{
a 846013116@qq.com

*Corresponding author: bjiangshuxia_2004@126.com.
} 
diameter, blade number, inlet installation Angle, distance between impeller and rear side plate, blade outlet width, volute width, volute tongue curvature, distance between impeller and socket tongue, noise.

In order to eliminate the influence of different dimensions on noise prediction, it is necessary to standardize the data. The standardization method adopted in this paper is shown in Equation (1):

$$
y=\frac{\left(y_{\max }-y_{\min }\right) *\left(x-x_{\min }\right)}{\left(x_{\max }-x_{\min }\right)}+y_{\min }
$$

Where, when the one-dimensional vector is normalized, $\mathrm{y} \max =1, \mathrm{y} \min =-1, \mathrm{x}$ is any sample point; X_min is the minimum sample point and $X \_\max$ is the maximum sample point. $\mathrm{Y}$ is the transformation data.

The normalized data were divided into training set and test set in a 9:1 ratio. The training set was used for the construction and training of the neural network model, and the test set was used for the evaluation of the neural network model.

\subsection{Multiple regression analysis and importance ranking of parameters}

Multivariate linear regression is one of the most commonly used analysis methods, which represents the relative linear relationship between a dependent variable and multiple independent variables. Based on this, the regression model among variables is established. Finally, the trend of the dependent variables is predicted through the regression parameters and errors. According to the influence of various parameters of the fan on noise, the mathematical model is established as follows:

$$
Y=\beta_{0}+\beta_{1} X_{1}+\beta_{2} X_{2}+\cdots+\beta_{16} X_{16}+\varepsilon
$$

Type: $\mathrm{Y}$ is noise, $X_{1}$ is flow rate, $X_{2}$ is total pressure, $X_{3}$ is the speed, $X_{4}$ is the power, $X_{5}$ is the efficiency, $X_{6}$ is the volute form, $X_{7}$ is the diameter of the impeller, $X_{8}$ is the diameter of the impeller inlet, $X_{9}$ is the diameter of the blade inlet, $X_{10}$ is the number of blades, $X_{11}$ is the installation Angle of the inlet, $X_{12}$ is the distance between the impeller and the rear side plate, $X_{13}$ is the width of the blade outlet, $X_{14}$ is the width of the volute, $X_{15}$ is the curvature of the socket tongue, $X_{16}$ is the distance between the impeller and the socket tongue, $\beta_{0}, \beta_{1}, \beta_{2}$. . . $\beta_{16}$ is a nonstandard regression coefficient, and $\varepsilon$ is a random error. According to the least square method, the non-standard coefficient can be obtained and then converted into the standard regression coefficient. The standard regression coefficient can be used to compare the influence degree of independent variable on dependent variable in the regression analysis. The greater the absolute value, the greater the influence degree. The conversion formula of non-standard regression coefficient and standard regression coefficient is as follows:

$$
\beta^{\prime}=\beta * \frac{S_{X}}{S_{Y}}
$$

In the formula, $\beta^{\prime}$ represents the standard regression coefficient, $S_{X}$ is the standard deviation of the independent variable and $S_{Y}$ is the standard deviation of the dependent variable. After transformation, the multiple regression equation is obtained as follows:

$$
\begin{gathered}
Y=1.629 X_{1}+1.148 X_{2}+0.404 X_{3}-1.438 X_{4}-0.303 X_{5} \\
-0.39 X_{7}+0.973 X_{8}+0.23 X_{9}+0.022 X_{10}+0.093 X_{11} \\
-0.265 X_{12}-0.121 X_{13}-0.815 X_{14}+0.242 X_{15} \\
+0.205 X_{16}
\end{gathered}
$$

It can be seen from the formula that the coefficient of $X_{1}$ is the largest, so the influence degree of flow rate on noise is the largest. The influence degree of each parameter on the noise is arranged from large to small: flow rate, power, total pressure, impeller inlet diameter, volute width, impeller diameter, efficiency, distance between impeller and rear plate, fossil-tongue curvature, blade inlet diameter, distance between impeller and fossiltongue, blade outlet width, inlet installation Angle, blade number, volute form.

\subsection{Determination of neural network topology}

When BP neural network is trained, too many hidden neural nodes will lead to overfitting of neural network and increase the training time. Too few implied neurons will lead to under-fitting of the network and reduce the accuracy of the network. In this paper, the optimal neuron node is determined by the combination of optimization and multiple regression. Main ideas as follows: the number of neurons in the input according to the results of the multiple regression sorted from 1 to 16 continuously accumulate, number of hidden neurons increased from 1 to 35 cycle, the output neurons to 1 , a total of 560 model, through the stand or fall of mean square error (MSE) evaluation model to select the most appropriate neuron nodes, the mean square error formula is:

$$
M S E=\frac{1}{n} \sum_{i=1}^{n}\left(y_{i}-\widehat{y}_{l}\right)^{2}
$$

Where $\mathrm{n}$ is the number of training samples and $y_{i}$ is the expected output value; $\widehat{y}_{l}$ is the network predicted value.

The smaller the MSE value is, the smaller the difference between the expected value and the predicted value in the state of the model is, indicating that the model has a better effect in the noise prediction of centrifugal fans. The larger the MSE value is, the difference between the expected value of fan noise and the predicted value of fan noise in the model state is too great, and the prediction effect is not good.

Table 1 shows the mean square error corresponding to different input neurons and implied neurons. It can be seen that when the input neuron is 4 and the implied neuron is 8 , the MSE is the smallest and the prediction effect of the neural network is the best.

Table 1. Different number of neurons corresponds to MSE value

\begin{tabular}{|c|c|c|c|c|c|c|}
\hline \multirow{3}{*}{ Input neuron } & \multicolumn{6}{|c|}{ Hidden neuron } \\
\cline { 2 - 7 } & 5 & 6 & 7 & 8 & 9 & 10 \\
\hline
\end{tabular}




\begin{tabular}{|c|c|c|c|c|c|c|}
\hline 3 & 1.13 & 1.01 & 0.81 & 0.53 & 4.69 & 4.15 \\
\hline 4 & 1.17 & 1.18 & 0.71 & $\underline{0.40}$ & 12.0 & 3.57 \\
\hline 5 & 0.65 & 1.09 & 0.57 & 0.93 & 4.46 & 2.59 \\
\hline 6 & 0.54 & 1.27 & 0.41 & 1.84 & 4.10 & 48.2 \\
\hline
\end{tabular}

\subsection{Improvement of particle swarm optimization algorithm}

Particle Swarm Optimization (PSO) is a widely used optimization algorithm, whose idea comes from the study on the predatory behavior of birds . In general, in the actual use of PSO algorithm, because particles always move towards the historical optimal position, the particle swarm will appear the phenomenon of convergence, easy to fall into local extreme value and premature convergence. In order to solve this situation, linear inertia weight decline is generally adopted. The inertia weight is shown in Equation (6).

$$
W=W_{\max }-\frac{\left(W_{\max }-W_{\min }\right) * t}{t_{\max }}
$$

Where, W_max is the maximum inertia weight; $\mathrm{W}$ _min is the minimum inertia weight; $\mathrm{T}$ is the actual number of algorithm runs; $T$ max is the maximum number of times the algorithm can run. In general, when W_max $=0.9$ and W_min $=0.4$, a better optimization effect can be achieved. The inertial weight $\mathrm{W}$ can control the convergence and search ability of the particle swarm optimization algorithm. The larger inertial weight enables the particles to move faster and have the ability of global search. Small inertial weight enables particles to have a small moving speed and the ability to search locally.

Linear decrease of inertia weight effectively improves the disadvantage of fixed linear weight of original particle swarm optimization algorithm, and realizes the decrease of linear weight from large to small, which can improve the searching ability of the algorithm to a certain extent. However, it may fall into the local optimal stage prematurely, resulting in the imbalance between global and local search capabilities. In this paper, the characteristics of inertia weight decline are used to make adaptive adjustments to $\mathrm{W}$ to reduce the probability that the algorithm falls into local optimum prematurely. The nonlinear decline strategy shown in Equation (7) is proposed to adjust the value of inertia weight $\mathrm{W}$. The curve of its inertia weight changing with the number of iterations is shown in Figure 1.

$$
W=W_{\text {min }}+\left(W_{\text {max }}-W_{\text {min }}\right) * e^{\left(-a *\left(\frac{t}{t_{\text {max }}}\right)^{3}\right)}
$$

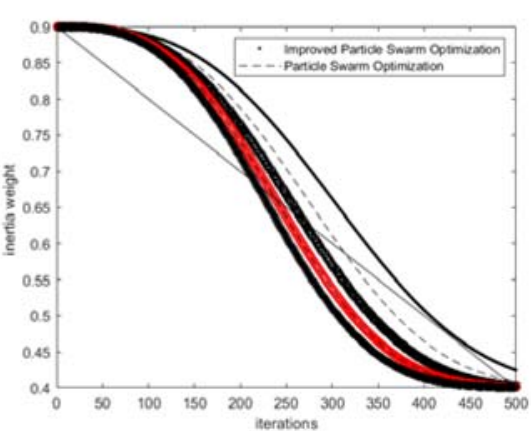

Figure 1. Change curve of optimal fitness

Type (7) a is adjusted factor, figure 1 shows the different values of a function of the curve, the figure shows that $\mathrm{a}=3$, the inertia weight the whole is greater than the linear decreasing weight, results in the decrease of the local search ability of particle, with the continuous increase of a value, the image down gradually, and the intersections of the linear weighting high constantly, constantly stronger local search ability. The stand or fall of a function of optimization algorithm, the key lies in whether can in global search and local search to find a more appropriate balance between, when $\mathrm{a}=6$, early algorithm can slow the rate of decreasing inertia weight, maintaining the larger value of for a long time, to explore more areas, and $\mathrm{W}$ in the late algorithm can dramatically reduce, Further improve the ability of local search, make the algorithm balance global search and local search ability is the strongest.

\section{Results and Analysis}

The improved PSO-BP neural network noise prediction model of centrifugal fan established in this paper adopts 4-8-1 structure. The input data of neuron nodes are flow rate, power, full pressure and impeller inlet diameter, and the fan noise is the output data. In this paper, the transfer function of hidden layer neurons is set as Tansig function through MATLAB software experiment. The neuron transfer function in the output layer was set as a linear function Purelin function, and the training function was set as trainlm function. 360 data in the training set were used for the training of the neural network model, and 40 data in the test set were used to test the accuracy of the neural network. The particle dimension $\mathrm{D}$ of the adaptive improved PSO algorithm is 43. When IPSO-BP neural network is trained, the maximum iteration time is 1000 , and the training target accuracy is 0.001 . The standard BP network, the BP network optimized by linear decreasing inertia weight PSO, and the BP network optimized by nonlinear inertia weight improved PSO were established respectively. The same training samples were trained respectively, and then 40 groups of test samples were input into the network. The optimal fitness change curve is shown in Figure 2. It can be seen that the convergence speed of linear decline weight is slow and the convergence result is large, while the fitness of the improved nonlinear decline weight is lower than that of linear decline weight, the convergence speed is fast and the convergence result is good. 


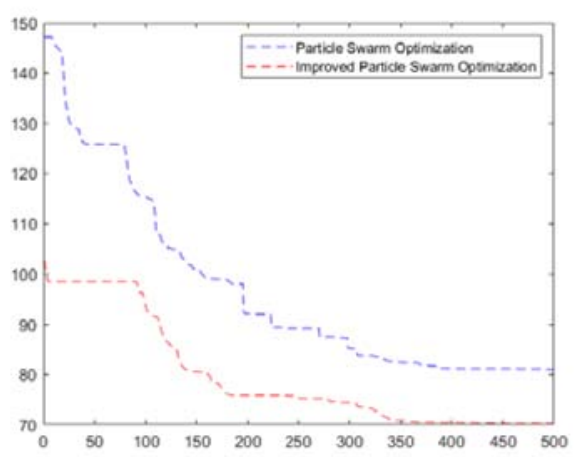

Figure 2. Change curve of optimal fitness

Table 2. Noise prediction errors of three models of centrifugal fans

\begin{tabular}{|c|c|c|}
\hline Prediction model & $\begin{array}{c}\text { Mean relative } \\
\text { error }\end{array}$ & $\begin{array}{c}\text { Maximum } \\
\text { relative error }\end{array}$ \\
\hline BP & $1.32 \%$ & $4.26 \%$ \\
\hline PSO-BP & $1.15 \%$ & $3.64 \%$ \\
\hline IPSO-BP & $0.97 \%$ & $2.92 \%$ \\
\hline
\end{tabular}

It can be seen from Figure 3 and Table 2 that the noise prediction models of these three centrifugal fans can predict the noise of centrifugal fans well, and the output error is not more than $2 \%$. Among the three prediction models, the standard BP neural network noise prediction model has the largest prediction fluctuation, and the prediction effect is slightly worse than the other two methods. Among the 40 test samples, 6 test samples have a relative error of more than $3 \%$, the maximum error is 4 . $26 \%$, and the average error is $1.32 \%$. The prediction accuracy of the BP neural network noise prediction model improved by PSO algorithm is improved, which indicates that the PSO algorithm optimizes the initial weight and threshold of BP neural network, accelerates the convergence of the neural network, and enhances its computing ability. Among them, the BP network centrifugal fan noise prediction model optimized by linear weight PSO has a relative error of more than $3 \%$ in 3 of 40 test samples, the maximum error is $3.64 \%$, and the average error is $1.15 \%$. The prediction model of IPSO-BP centrifugal fan noise adopted in this paper has high prediction accuracy, and the prediction results are relatively stable. The maximum error is reduced to 3.72 . In 40 test samples, the error of all samples is less than 3\%, and the average error is also reduced to $0.97 \%$.

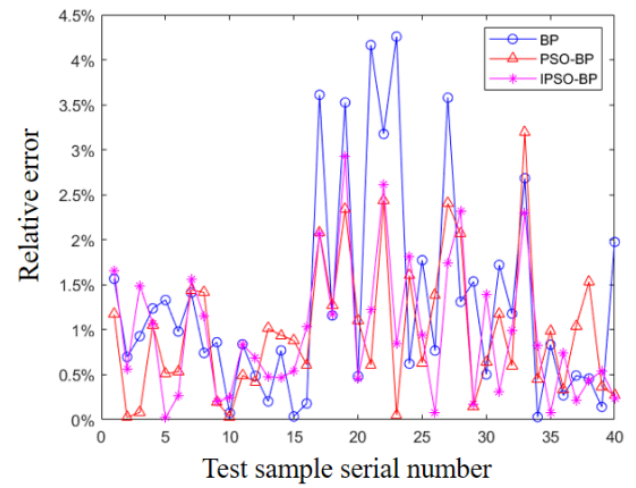

Figure 3. Identification accuracy and error comparative analysis

\section{Conclusion}

Based on traditional BP algorithm easy to fall into local optimum, a good global search ability is improved, meet using the improved PSO algorithm to find the optimal threshold, the initial weights of BP network by using regression analysis of the parameters of the fan is importance analysis, combining with the neural network optimization algorithm to determine the best input neurons and the hidden neurons of centrifugal fan noise prediction. The results show that compared with the previous two models, the prediction model adopted in this paper reduces the possibility of falling into the local extreme value, speeds up the convergence speed, improves the accuracy and reliability of the prediction of centrifugal fan noise, and can meet the requirements of the prediction of centrifugal fan noise.

In this paper, based on IPSO - centrifugal fan noise prediction model of BP neural network can realize effective prediction of centrifugal fan noise, by means of centrifugal fan noise prediction, forecast the centrifugal fan noise is in line with the environmental protection as well as the customer request, reduces the noise when centrifugal fan production does not conform to the requirements to be returned to the factory to remake the manpower and financial resources. The method can also be applied to axial flow fan and mixed flow fan, and can be improved for other fields.

\section{Acknowledgments}

Natural Science Foundation of Hunan Province, No. 2019JJ60076

\section{References}

1. Yao J Y. Aerodynamic noise prediction of tube-axial fan based on BP artificial neural network[D]. Nanjing university, 2020.

2. Wei Z, Deng Y C, Yang Z H. Wind farm noise prediction model based on Elman neural network[J]. Hydroelectric energy science, 2015, 33(05):203-206.

3. Hua C L, Bi T F, Wang J X, Internal noise prediction based on neural network tools $[\mathrm{J}]$. Automotive engineer, 2016(04):26-30.

4. Li Y G. Prediction of CPU voltage noise based on BP neural network[J]. Electronic Design Engineering, 2018, 26(23):169-172+177.

5. Li H, Xiao X B, Jin X S. Aerodynamic noise prediction of high-speed train based on neural network method[J]. Noise and vibration control, 2015, 35(03):56-59+116.

6. Cheng J. Wind blower noise prediction based on regression analysis and BP neural network[J]. Noise and vibration control, 2013, 33(06):49-52+123. 